\title{
Estimación de la carga máxima de sedimentos para la determinación de la formación de bancos de arena sobre el Río Cauca, Jamundí-Valle del Cauca
}

\section{Estimation of the maximum load of sediments for the determination of the formation of sand banks on the Cauca River, Jamundi-Valle del Cauca}

\author{
Eduard Paz Zúñiga \\ Ingeniero Topográfico, Universidad del Valle. Cali-Colombia. \\ eduardfpaz@gmail.com | 0000-0002-2654-1839
}

\begin{abstract}
Para citar este artículo: Paz Zúñiga, E. (2021). Estimación de la carga máxima para la determinación de la formación de bancos de arena sobre el río Cauca, Jamundí - Valle del Cauca. Entorno Geográfico, (21), 1-20. https://doi.org/10.25100/eg.v0i21.11306
\end{abstract}

\section{Resumen}

La estimación de la carga máxima de sedimentos sobre el lecho de río permite determinar las zonas de acumulación y posterior formación de bancos de arena, en este artículo se presentan los resultados de la estimación de la carga máxima de sedimentos sobre el lecho del Río Cauca. La zona de estudio se caracteriza por presentar un gradiente bajo y de forma sinusoidal, además de una climatología tropical de solo dos estaciones, donde se tienen temporadas de lluvia en la que se da el transporte, seguidas de calurosas épocas de verano donde se acumula el material de arrastre sobre el lecho del río, características suficientes para la formación de bancos de arena. Se partió de estudios realizados en ríos con características fluviales y topográficas muy parecidas a las de la zona de estudio, los cuales, a partir de la aplicación de modelos matemáticos a los valores de pixel de las fotografías aéreas del río, permitieron el reconocimiento de cauce y formación de bancos de arena sobre el lecho. Para el cálculo de transporte de sedimentos o carga de fondo se utilizó la relación de Meyer- Peter \& Muller (Ferguson, 2008) y la función de Shields, Church (2006), modelos que dependen de las características topográficas del lecho del río. Se utilizó el modelo digital de elevación obtenido del procesamiento fotogramétrico, así mismo, se estableció un tamaño de grano de $4 \mathrm{~mm}$ y una velocidad virtual de $1.3 \mathrm{~m} / \mathrm{s}$, para el reconocimiento definitivo del lecho se optó por las funciones de potencia y tensión de corte o erosión Lorang et al. (2005). Los resultados obtenidos mostraron que el Río Cauca tiene gran capacidad de acumulación, al igual que de transporte de sedimentos, se destacaron zonas de 
acumulación, que para el año de estudio presentaban un potencial para la formación de bancos de arena, los que a la fecha continúan siendo explotados, los resultados de carga de fondo, formación de bancos de arena y reconocimiento del lecho de río, mostraron coherencia entre sí, permitiendo tener validez en los resultados, para un río de las características estudiado.

Palabras clave: Fotografías aéreas, Transporte de sedimentos, Bancos de arena, lecho de río, Modelo digital de elevación.

\begin{abstract}
The estimation of the maximum load of sediments on the river bed allows to determine the zones of accumulation and later formation of sand banks. This article presents the results of the estimation of the maximum load of sediments on the river bed of the Cauca River. The study zone is characterized by a low gradient and sinusoidal shape, in addition to a tropical climate of only two seasons, where there are rainy seasons in which the transport occurs, followed by hot summer seasons where the dragging material accumulates on the river bed, characteristics sufficient for the formation of sand banks. We started from studies carried out in rivers with fluvial and topographic characteristics very similar to those of the study zone, which, from the application of mathematical models to the pixel values of the aerial photographs of the river, allowed the recognition of the riverbed and the formation of sand banks on the bed. For the calculation of sediment transport or bottom load, the relationship of Meyer-Peter \& Muller (Ferguson, 2008) and the function of Shields, Church (2006) were used, models that depend on the topographical characteristics of the river bed. The digital elevation model obtained from photogrammetric processing was used, as well as a grain size of $4 \mathrm{~mm}$ and a virtual speed of 1.3 $\mathrm{m} / \mathrm{s}$. For the definitive recognition of the bed, the power and shear stress or erosion functions were chosen by Lorang et al. (2005). The results obtained showed that the Cauca River has great accumulation capacity, as well as sediment transport. Accumulation zones were highlighted, which for the year of study presented a potential for the formation of sand banks, which to date continue to be exploited, the results of bottom loading, sand bank formation and river bed recognition, showed coherence between them, allowing validity in the results, for a river of the characteristics studied.
\end{abstract}

Keywords: Aerial photographs, Sediment transport, Sandbanks, Riverbed, Digital elevation model.

Recibido: 24 de febrero de 2020

Aceptado: 04 de agosto del 2020 


\section{Introducción}

Los ríos son la fuente natural más sobre explotada por el sector constructivo, los diferentes usos y las excelentes propiedades físico-mecánicas como agregados para la construcción hacen que tanto las arenas como gravas sean muy apetecidas por el mercado, la continua extracción de material con dragas y de manera artesanal con malacates han logrado que la morfología del lecho del río se vea afectada, aguas arriba como aguas debajo de los puntos y/o sectores de extracción.

De acuerdo con Ramírez et al. (2009), la carga máxima de sedimentos que posee un río permite establecer cuál será la cantidad de material disponible anualmente en los bancos de arena depositados luego del arrastre (CAMACOL, 2013). La identificación correcta de puntos de extracción permite además de conocer la oferta de materiales de arrastre, establecer controles ambientales partiendo de la capacidad métrica de acumulación anual que posee el lecho del río. Según la Corporación Autónoma del Valle del Cauca CVC (1999) la extracción de materiales de arrastre no debe superar el 25\% de acumulación anual en temporadas de verano o sequía, no obstante, se reconoce que debido a la demanda de materiales de arrastre se puede superar esta tasa de extracción siempre y cuando no se afecte la morfología del río.

El Río Cauca se caracteriza por ser del tipo sinusoidal y de acumular material de arrastre en las zonas internas de curvas pronunciadas sobre la zona del Valle del Cauca. Conocer los sectores de acumulación y estimar la carga máxima, permiten establecer la disponibilidad de materiales para ser utilizados en el sector de la construcción y el desarrollo de la infraestructura de una ciudad.

En este proyecto se estimó la carga máxima de sedimentos del Río Cauca, en el tramo de Jordanes - Paso de la Bolsa - Bocas de Palo, Jamundí, Valle del Cauca, para determinar la formación de bancos de arena y afectación del lecho del río. En el procesamiento y para la obtención de resultados se trabajó inicialmente en el procesamiento fotogramétrico, del cual se obtuvieron productos como el Modelo digital de elevaciones DEM y el fotomosaico. Posteriormente se realizó el procesamiento y análisis, priorizando el lecho del río como zona de interés para la visualización, análisis de resultados y generación de salidas gráficas.

El DEM y fotomosaico, permitieron estimar la carga máxima de sedimentos partiendo de la pendiente del lecho y la longitud de acumulación con la relación de Meyer- Peter \& 
Muller según Ferguson (2008) y la función de Shields según Church (2006). Al visualizar los resultados y reconocer los valores establecidos para que el material de arrastre se acumule naturalmente y forme los bancos de arena, se presentaron los resultados de carga de fondo y capacidad de formación de bancos de arena o acumulación de sedimentos.

Adicionalmente se reconoció el lecho del río a partir de factores como la potencia y la tensión de corte (erosión) Lorang et al. (2005), la combinación de los tres productos permitió estimar los sectores de mayor capacidad de acumulación de sedimentos e identificar aquellos con más daños causados por la sobre explotación de materiales de arrastre, lo que permitirá posteriormente la elaboración de planes de contingencia y protección ambiental, así como también la designación de sectores de extracción de materiales de arrastre, por la entidades interesadas.

\section{Materiales y métodos}

\section{Zona de estudio}

El presente proyecto de investigación se desarrollará sobre el Río Cauca en el sector de Jamundí, Valle del Cauca, refereido por el tramo Jordanes-Paso de la Bolsa-Bocas, ver (Figura 1). Este tramo es el límite natural entre los departamentos del Cauca y Valle del Cauca, el cual se caracteriza por ser uno de los sectores más sobre explotados gracias a la fácil accesibilidad que provee la carretera panamericana y la constante disponibilidad de material de arrastre (Corporación Regional del Valle del Cauca-CVC-, 2008). El paisaje corresponde al de valle aluvial de Río Cauca con altura promedio de 960 msnm. 
Fuente: Google Earth.

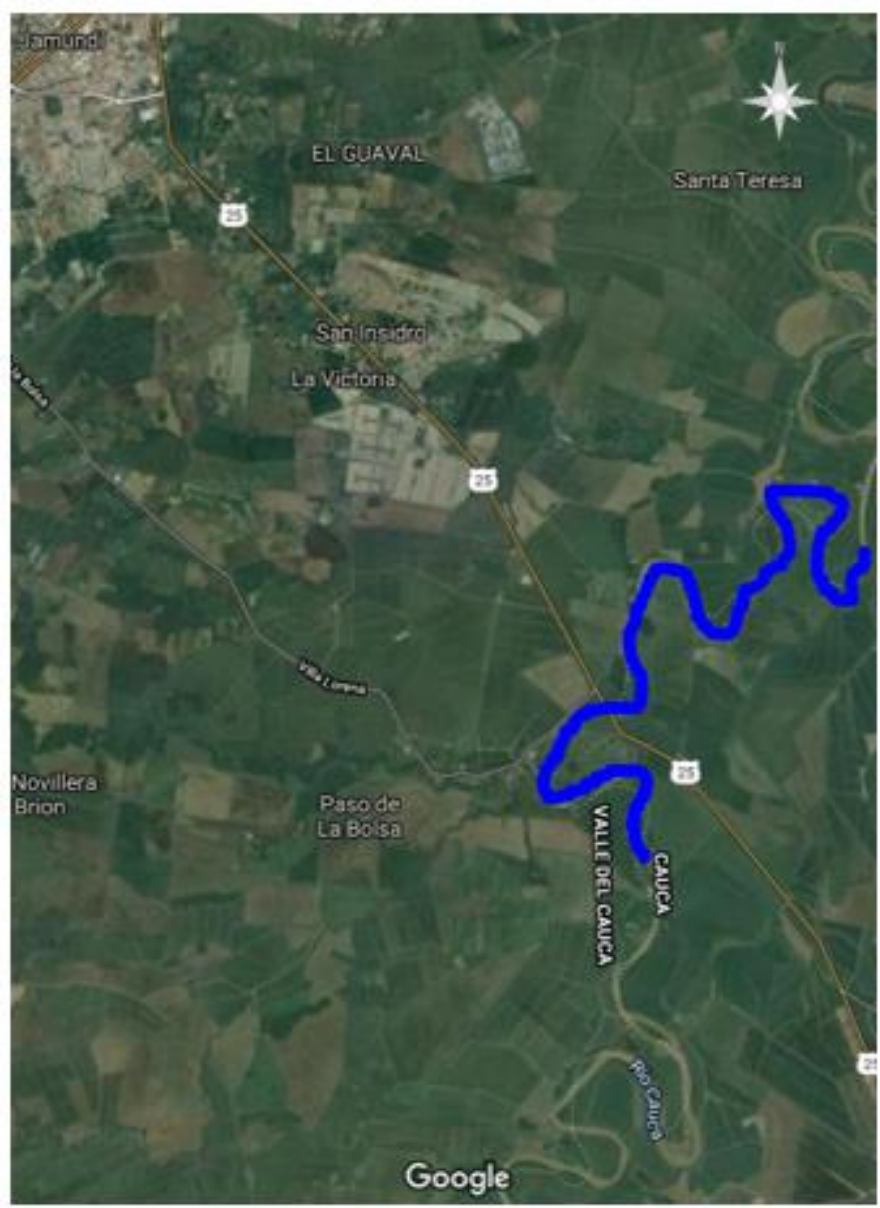

Figura 1. Localización del tramo Jordanes-Paso de la bolsa-Bocas de palo. Jamundí, Valle del Cauca.

\section{Fuentes de información}

Fueron analizadas 5 fotografías aéreas suministradas por la división de Planoteca y Cartografía de la CVC, las cuales corresponde a el área de estudio antes nombrada, estas fueron tomadas por la firma FAL, en el año de 1999 debido a una inundación registrada en ese mismo año. Las fotografías corresponden al vuelo 415 y Faja 02, desde la fotografía 81 hasta la 85 (Figura 2), las cuales se tomaron con una altura de vuelo de $2444.2 \mathrm{~m}$, configurando una escala de trabajo de 1:10200.

Además de las fotografías aéreas se utilizó Google Maps en la opción de satélite, para la discusión y análisis de resultados. 


\section{Pre-procesamiento}

Para realizar el procesamiento de los productos se adelantó inicialmente:

\section{Obtención de puntos de control}

Fuente: Elaboración propia.

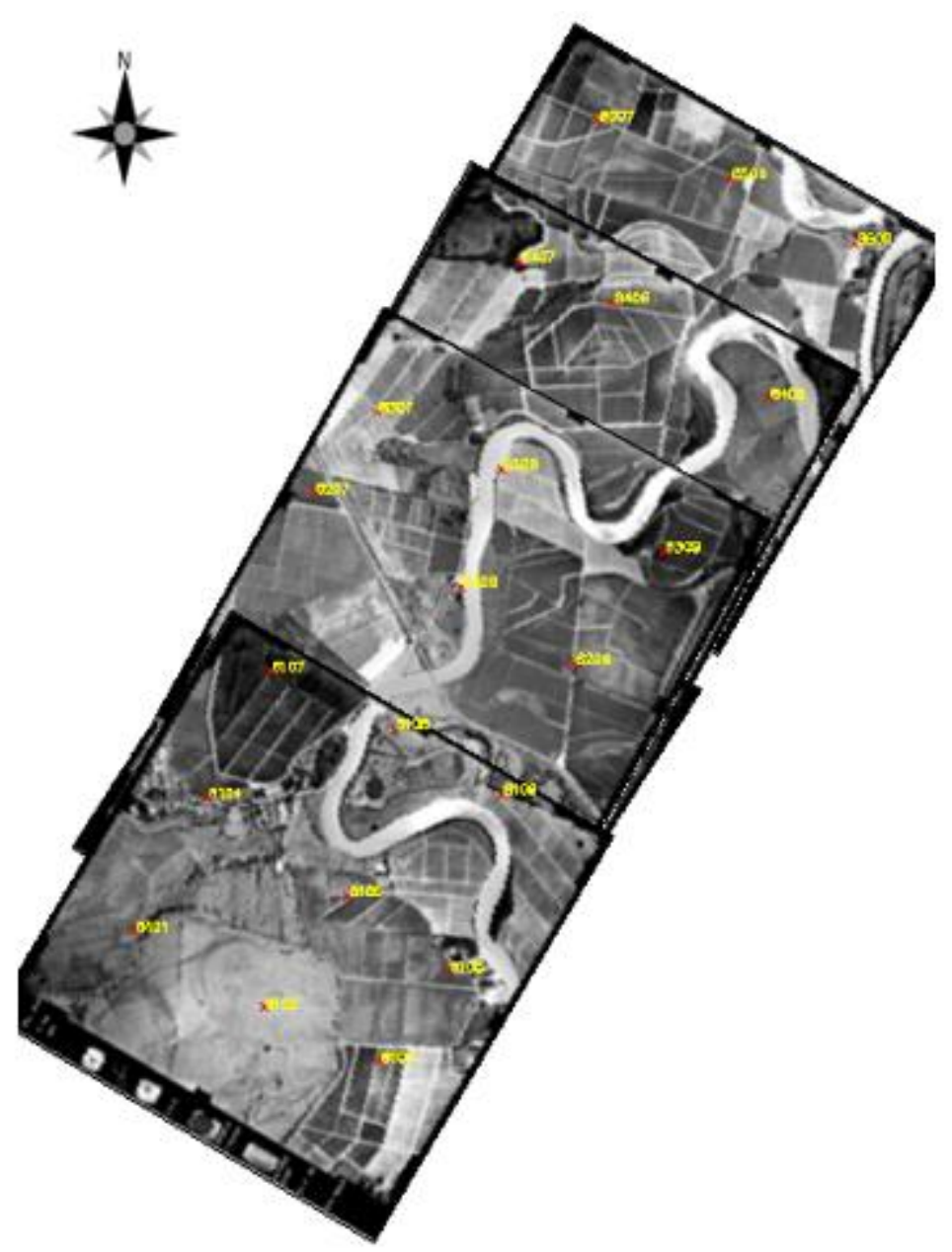

Figura 2. Puntos de control sobre las fotografías aéreas. Fotografías FAL-415F02, 81-

85 .

Por medio de la herramienta de satélite de Google Maps y contando con muy buena resolución de detalle $(<5 \mathrm{~m})$ para la zona de estudio se tomaron un total de 21 puntos de control distribuidos homogéneamente en todas las fotografías, los cuales fueron transformados del sistema de referencia origen Bogotá a Magna SIRGAS oeste, para generar la posterior triangulación. A los puntos de control le fueron asignados un número de secuencia a partir del número de fotografía donde se encontraban. 


\section{Procesamiento fotogramétrico}

Para la generación y aplicación de los modelos, se partirá de los procesos fotogramétricos elementales de generación de los mismos, tales como:

\section{Bloque de procesamiento}

Se parte de la creación del bloque mediante la herramienta LPS del software ERDAS 9.2, con las propiedades iniciales de altura de vuelo, modelo de la cámara, marcas fiduciales y coordenadas de estas (Tabla 1).

Tabla 1. Datos utilizados para la generación del bloque.

\begin{tabular}{|c|c|}
\hline Sistema de referencia & Referencia de la cámara \\
\hline $\begin{array}{l}\text { - Tipo de Proyección: } \\
\text { Transverse Mercator } \\
\text { - Origen: Colombia West } \\
\text { Zone } \\
\text { • Esferoide Horizontal: WGS } \\
84 \\
\text { - Factor de Escala Meridiano } \\
\text { Central 1.000 } \\
\text { - Longitud: } 77^{\circ} \\
04^{\prime} 39,02 \text { ', W } \\
\text { - Latitud: } \\
\text { 35'46.32', N } \\
\text { - Falso Este y Norte: } \\
\text { 1000000.000 } \\
\text { - Esferoide y Datum } \\
\text { Vertical: Wgs } 84\end{array}$ & $\begin{array}{l}\text { - Cámara: RMK A 15/23 } \\
\text { - Longitud Focal: } 152.914 \mathrm{~mm} \\
\text { - Punto Principal: Xo= }-0.009 \text { Yo = } \\
-0.004 \\
\text { - Fiducial 1: X1 112.993 Y1 } \\
0.001 \\
\text { - Fiducial 2: X2 -113.003 Y2 } \\
\text { 0.006 } \\
\text { - Fiducial 3: X3 -0.011 Y3113.014 } \\
\text { - Fiducial 4: X4 -0.006 Y4 - } \\
\text { 112.994 } \\
\text { - Altura del Vuelo: } 2444.1912 \mathrm{~m} \\
\text { (8019 pies) }\end{array}$ \\
\hline
\end{tabular}

Fuente: Elaboración propia.

\section{Orientación Interna y Externa}

La orientación interna es el proceso que permite reconstruir el haz de rayos de una imagen para corregir la geometría en el instante de la toma, por lo cual, este procedimiento permite recuperar la geometría original a partir de las marcas fiduciales, para el caso fueron utilizadas las descritas por el certificado de calibración (Zeiss, 1996) y capturadas 
en el siguiente orden (Figura 3). Las coordenadas de los puntos fiduciales insertados se transformaron del Sistema de Coordenadas interno de la cámara al Sistema de Coordenadas píxel utilizando el módulo LPS del software ERDAS. Posteriormente se realizó el ajuste de mínimos cuadrados de las 4 marcas fiduciales en cada una de las fotografías que se quieren utilizar en el proyecto, los errores se mantuvieron por debajo de 0.3 pixel en esta fase.

Fuente: Certificado de calibración (1996).
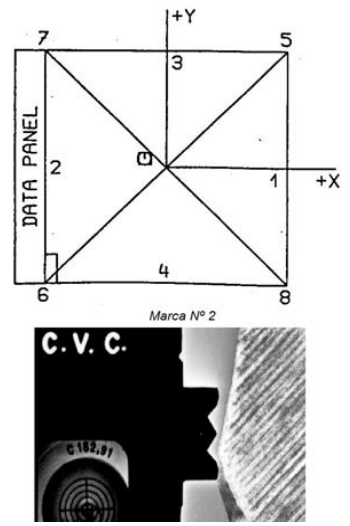

Figura 3. Arriba: Numeración de las marcas fiduciales. Abajo: Detalle de marcas fiduciales utilizadas.

Haciendo uso del módulo LPS del software ERDAS, se realizó el proceso de orientación externa para determinar la posición de las imágenes en el espacio (Figura 4), utilizando los 21 puntos de control, que cubren totalmente el área de las fotos.

Fuente: Elaboración propia.

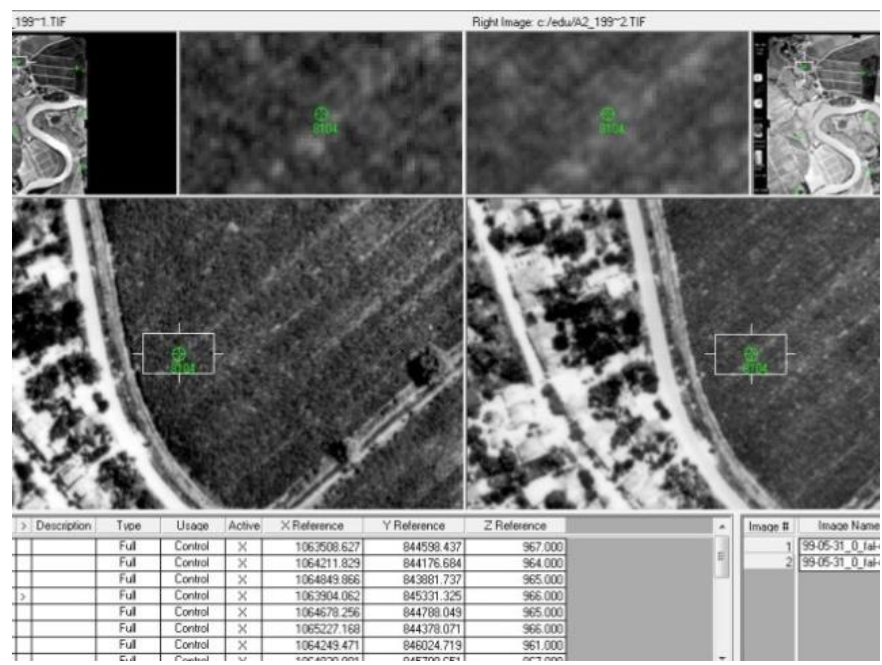

Figura 4. Orientación externa, utilizado del módulo LPS del software ERDAS. 


\section{Aero triangulación y puntos TIE}

Para el pre procesamiento, la aero triangulación automática sobre las cinco fotografías aéreas se encarga del amarre físico y matemático en bloque de fotografías individuales determinando de forma indirecta sus parámetros de orientación y asociando todo el proyecto al Datum de referencia horizontal y vertical.

En este procedimiento se logró conseguir un RMSE inferior a 0.3 unidades, obteniendo un error de 0.2790 pixel. Al realizar el proceso de creación de puntos TIE, se obtuvo la triangulación final lo que permitió la correcta generación de productos como el modelo de elevaciones, las ortofotos y finalmente el mosaico.

\section{Procesamiento de los modelos}

Una vez obtenido todo el pre-procesamiento sobre las fotografías se estimó la carga máxima de sedimentos. Para el cálculo, se realizó un recorte del modelo de elevaciones el cual comprende el espejo de agua y bordes de terreno, tomando como limite el lecho de río y sobre esta nueva capa se realizaron los cálculos de la carga máxima de sedimentos o transporte de sedimentos que se obtuvo a partir de variables topográficas como la pendiente del lecho y longitud de acumulación; el cálculo de la carga de fondo se realizó a partir de la relación de Meyer- Peter \& Muller (Ferguson, 2008).

$$
Q_{S} \propto w \tau_{c}^{1.5}\left(\frac{\tau}{\tau_{c}}-1\right)^{1}
$$

Donde $\tau \propto d S, \tau_{C} \propto D$ y $w, d, S, D$ corresponde al ancho, profundidad, pendiente y al diámetro medio del tipo de grano, respectivamente. Si se quiere tener una mejor representación de los resultados se puede optar por los escudos de Shields donde se debe partir de la relación de Lane's que dispone descarga $(\mathrm{Q})$ gradiente $(\mathrm{S}) \sim \mathrm{QbD} / \mathrm{d}$, en la que Qb es la carga material del lecho y d es profundidad del cauce, por lo cual al reorganizar tenemos finalmente. La función de Shields,

$$
\frac{Q b}{Q}=f\left[\frac{\rho g d_{s}}{g(\rho s-\rho) D}\right] \therefore \tau^{*}=[(\rho s-\rho]
$$

La anterior ecuación representa el transporte de sedimentos, donde $\rho s$ y $\rho$ son la densidad del sedimento y el agua respectivamente, $g$ la aceleración de la gravedad, d la profundidad del cauce y D es el tamaño del grano a mover. Este modelo es sustentado a partir de la relación de Meyer-Peter \& Muller (Ferguson, 2008), lo que permite realizar una 
comparación de los resultados obtenidos por cualquiera de los dos métodos y disponer de un modelo promedio de dispersión de los resultados.

La acumulación sobre el lecho se da cuando la relación entre D/d > 0.1, lo cual determina la formación de bancos de arena o de acumulación de sedimentos.

La modelación del potencial del río se realizó a partir de la potencia de la corriente $(\mathrm{P})$, dependiendo de la pendiente (S), profundidad del agua (h) y la velocidad de flujo (V)

$$
P=\rho g h S V
$$

Donde $\rho$ es la densidad del agua y $g$ la gravedad promedio de la tierra. La tensión de corte (T) o erosión se mide como el aumento del cauce debido al aumento de la velocidad de flujo de la siguiente manera,

$$
T=\rho g h S
$$

Por lo tanto, si la profundidad del agua y la pendiente aumentan, así también lo hace el potencial del río para el movimiento de sedimentos. La combinación de la pendiente, profundidad y la velocidad de flujo proporcionan el conocimiento del potencial o poder a lo largo del cauce.

\section{Resultados}

\section{Modelo de elevación digital DEM}

La generación del modelo digital de elevación se realizó con el módulo LPS del software ERDAS, del cual se obtuvo tanto el modelo como la calidad del resultado obtenido. La generación del modelo realizo con un tamaño de pixel de 3 metros y teniendo en cuenta que el resultado final fuera una sola imagen (Figura 5).

Fuente: Elaboración propia.
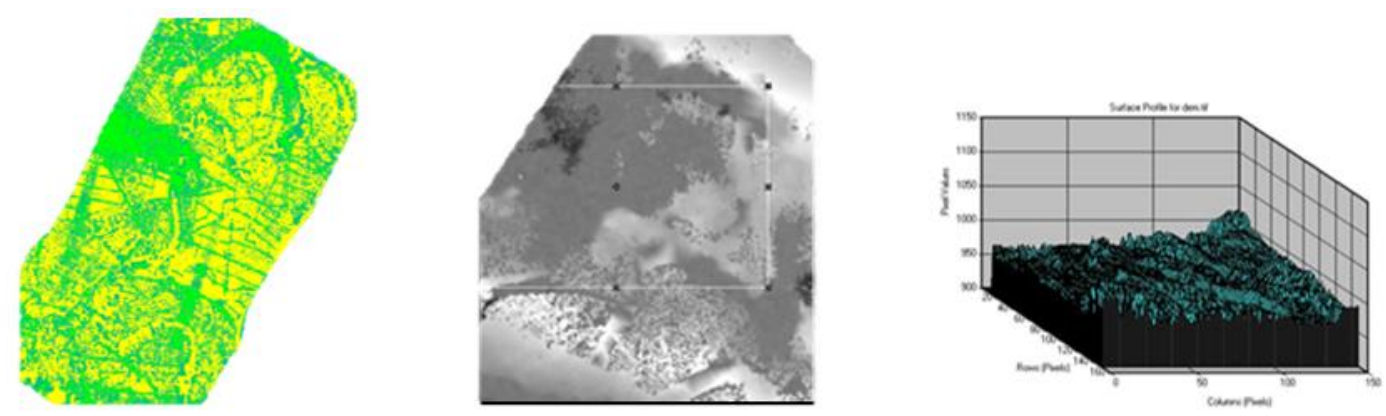
Figura 5. Izquierda. Calidad del DEM, Centro, visualización del DEM, Derecha.

Detalle del DEM.

\section{Mosaico}

Para completar el análisis se crea un mosaico a partir de las imágenes aéreas de la zona de estudio, orientadas externamente dentro del bloque. Esto se hace mediante la herramienta Mosaic Tool de Erdas, de la que se escogió la mejor forma de representación del mosaico, a partir del DEM en el paso anterior, obteniendo el resultado indicado en la Figura 6.

Fuente: Elaboración propia.

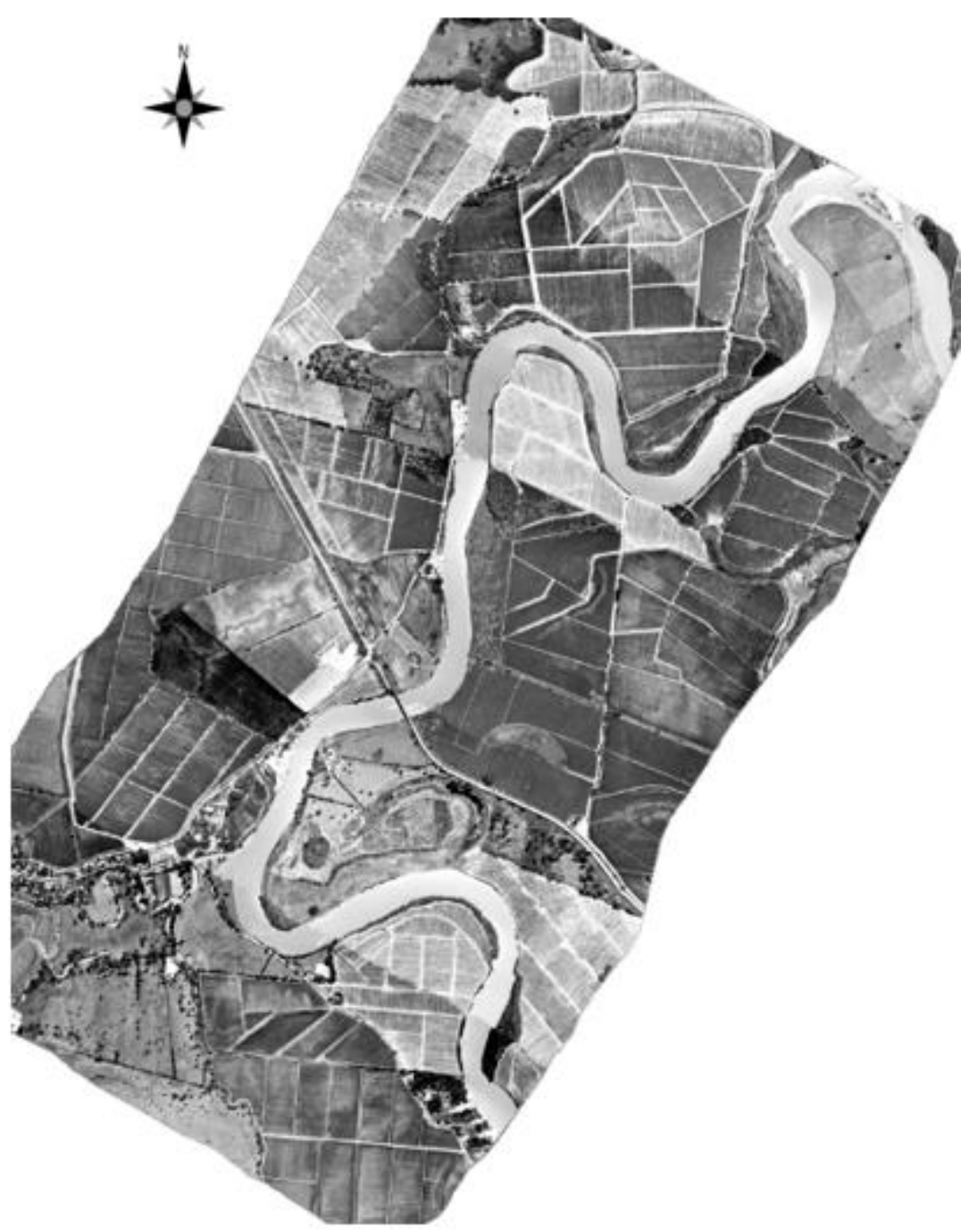

Figura 6. Mosaico generado. 


\section{Aplicación de los modelos}

Una vez obtenido el procesamiento sobre las fotografías y generados los insumos de inicio, que son el DEM y el fotomosaico, se estimó la carga máxima de sedimentos partiendo de la pendiente del lecho y longitud de acumulación.

El primer resultado se obtuvo al ejecutar el proceso fotogramétrico, el cual muestra la carga de fondo (capacidad de arrastre) o la fuerza de transporte de sedimentos sobre el lecho del río ver (Figura 7), cabe resaltar que la relación de Meyer- Peter \& Muller y la función Shields, son funciones que depende de variables muy similares, por los cual los resultados obtenidos no mostraron mayor dispersión.

Fuente: Elaboración propia.

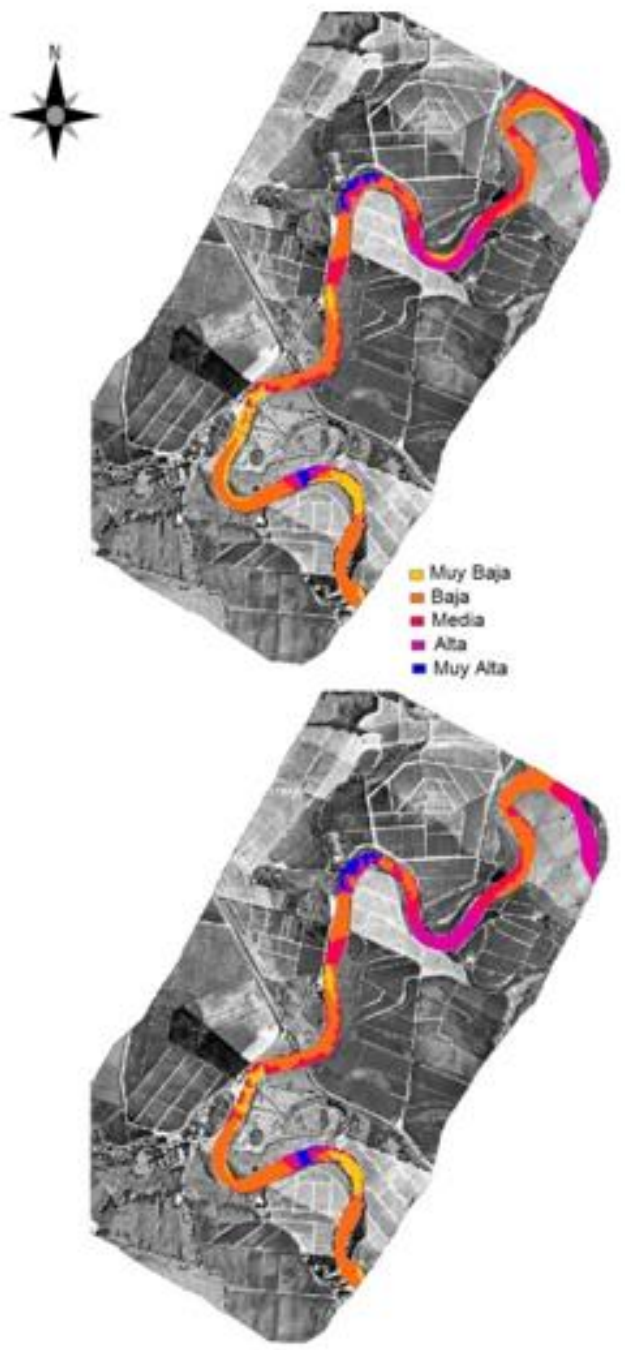


Figura 7. Cálculo de la carga de fondo. Arriba. Obtenido con la relación de MeyerPeter \& Muller. Abajo: Obtenido con la función Shields, el cálculo se realizó para un tamaño de grano 4mm (arena gruesa) areneros balastro o balastrillo.

Una vez obtenido el modelo de transporte de sedimentos sobre todo el lecho del río, se estimó la formación de bancos de arena o acumulación de sedimentos sobre el mismo teniendo en cuenta los resultados de valor de pixel antes obtenidos (Figura 8), se aprecia en tonos claros las zonas potenciales para la formación de bancos de arena.

Fuente: Elaboración propia.

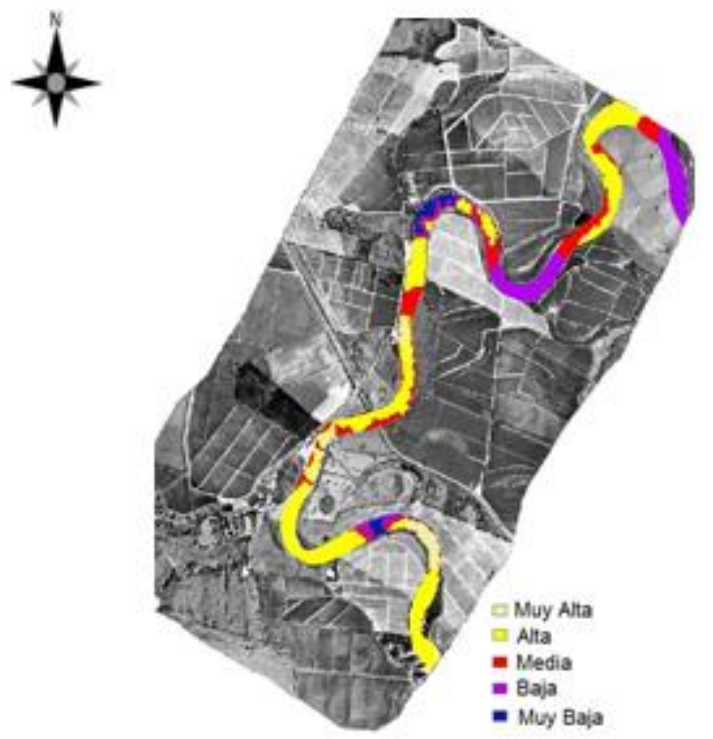

Figura 8. Capacidad de formación de bancos de arena.

Una vez caracterizados los sectores de acumulación, partiendo de los resultados del transporte de sedimentos, se reconoció el lecho del río, a partir de la aplicación a nivel de pixel de las funciones Potencia del Cauce y Tensión de Corte (Figura 9). Los resultados obtenidos mostraron coherencia y permitieron validar lo anteriormente encontrado. 
Fuente: Elaboración propia.
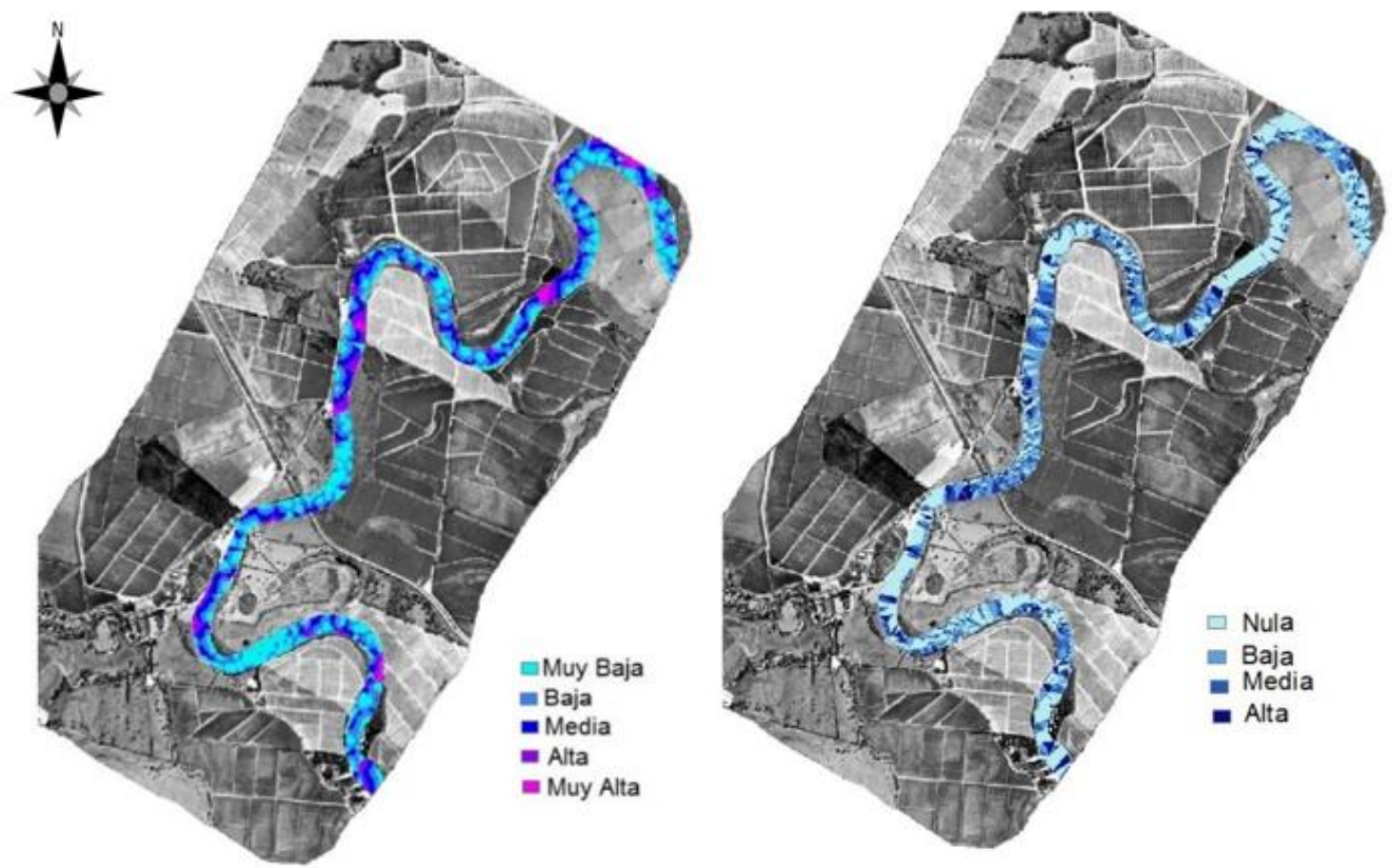

Figura 9. Reconocimiento del lecho del Río. Izquierda. Potencia del Río, Derecha.

Tensión de corte, el cálculo se realizó con una Velocidad virtual, de $1.3 \mathrm{~m} / \mathrm{s}$.

\section{Análisis}

\section{Pre-procesamiento}

Al realizar el proceso fotogramétrico se tuvieron muchas inconsistencias en la técnica como se capturaban los puntos de control sobre las fotografías y al correr la triangulación se presentaban errores muy altos, así que se optó por ir triangulando fotografía a fotografía y mientras el error se mantuviera por debajo de lo permitido se continuaba y se trataba de mantener la permisividad del error. Al finalizar cuando se triangulo en conjunto con las cinco fotografías se obtuvo un error de 0.2790 pixel, muy pequeño comparado con el obtenido anteriormente realizando la triangulación sin ir corrigiendo el error por cada fotografía anexada a la triangulación. 


\section{Resultados gráficos}

Los resultados obtenidos mostraron validez y coherencia entre sí, estableciendo una relación entre los modelos matemáticos utilizados en el lecho del río y el análisis a nivel de pixel.

Se muestra que ambas funciones (1) (2) al expresarse de manera similar generaron resultados muy parecidos, teniendo poca dispersión en los resultados generados (Figura 10). Se reconocen los colores cálidos, amarillo y naranja, por tener un gradiente bajo, por lo cual la carga de fondo también los es, lo que permite la acumulación de sedimentos y como se evidenciará más adelante la formación de bancos de arena (Figura 11). Una vez calculado el transporte de sedimentos o carga de fondo. La acumulación sobre el lecho se presenta cuando la relación entre $\mathrm{D} / \mathrm{d}>0.1$, lo que determina la formación de bancos de arena.

Fuente: Elaboración propia.

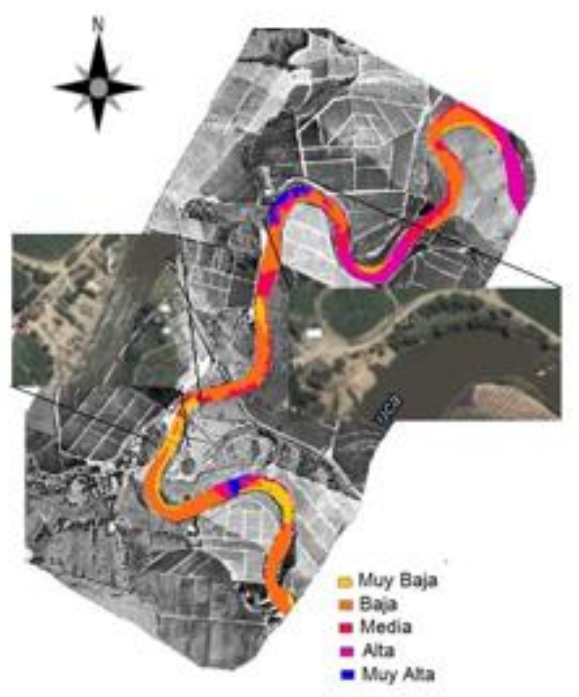

Figura 10. Relación entre los resultados obtenidos de transporte de sedimentos y las visualizaciones actuales de Google Earth.

La formación de bancos de arena de una u otra manera es el producto inverso del transporte de sedimentos, tal como se evidencia en los resultados esto se obtiene de manera comparativa, los lugares donde el transporte es alto, produciendo erosión, la acumulación es nula, mientras en las zonas donde el transporte es bajo y el lecho permite la acumulación se da la formación de bancos de arena o acumulación de sedimentos. En 
la Figura 11 se observa de colores claros y amarrillo las zonas potenciales de agregados o bancos de arena para la construcción siendo estas las posibles zonas de explotación.

Fuente: Elaboración propia.

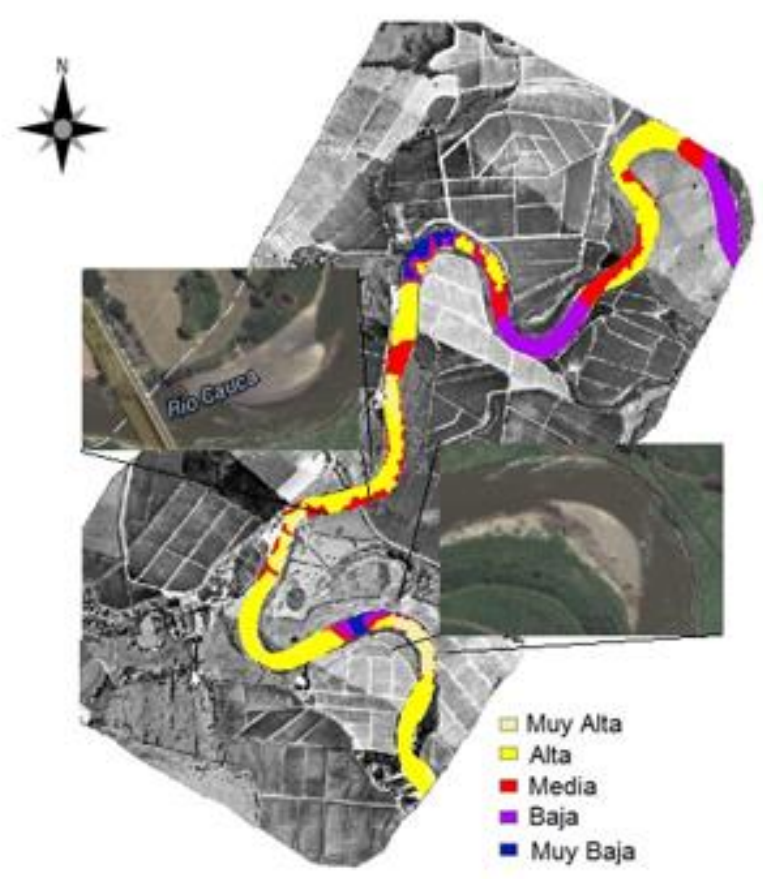

Figura 11. Relación entre los resultados obtenidos de formación de bancos de arena y las visualizaciones actuales de Google Earth.

Realizando la comparación de la situación actual basada en la visualización de Google Earth v/s los resultados obtenidos, se muestra validez gráfica, ya que las zonas potenciales para la formación de bancos de arena para la fecha de captura 1999 como se evidencia a la fecha 2014 presentan una gran acumulación de sedimentos con relación a la formación de bancos de arena. Para el caso de transporte de sedimentos se ve gran relación respecto al transporte bajo de sedimentos y las zonas actuales de explotación artesanal, ya que esta captura los sedimentos que están próximos a la cama o lecho del río, en relación a esto se destaca la zona ubicada al sur del puente de la vía panamericana que atraviesa el río Cauca, ya que esta es una de las zonas actualmente sobre explotadas y para la fecha de captura muestra el completo potencial para la acumulación y generación de bancos de arena debido a un transporte de sedimentos bajo (Figura 12). 
Fuente: Elaboración propia.

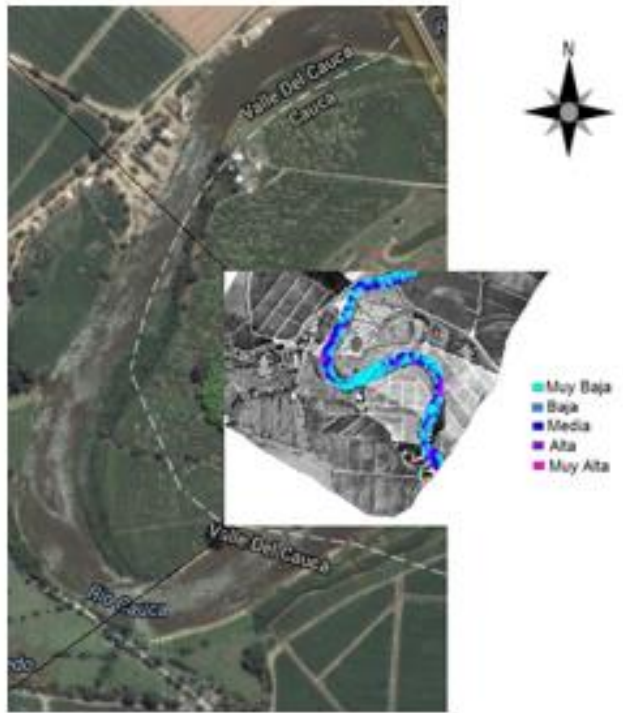

Figura 12. Relación entre los resultados obtenidos de potencia y las visualizaciones actuales de Google Earth.

Los resultados del reconocimiento del lecho de río a partir de la potencia y tensión de corte validan las premisas antes obtenidas, ya que como se puede observar, la potencia del río se caracteriza por tomar las zonas externas en las curvas del río, lo que indica que en estas zonas hay mayor velocidad y por ende mayor transporte de sedimentos. En relación con el estado actual, en la Figura 13 se ve la coincidencia de la potencia media obtenida por el procesamiento en tonos de azul y los colores claros de aumento del caudal evidenciados en la imagen de Google Earth. 
Fuente: Elaboración propia.
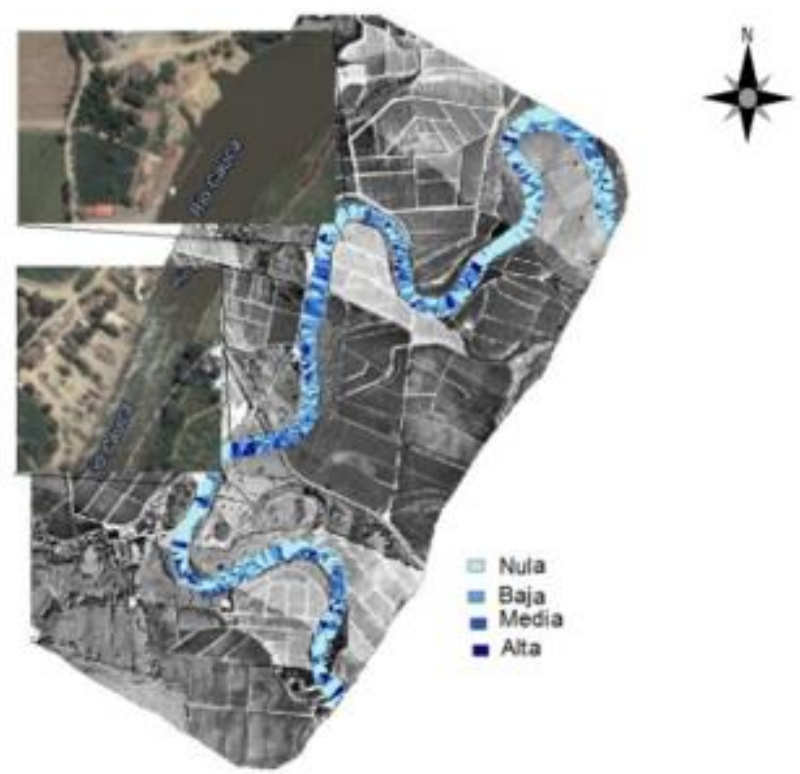

Figura 13. Relación entre los resultados obtenidos de tensión de corte o erosión y las visualizaciones actuales de Google Earth.

Respecto a la tensión de corte o zonas afectadas por la erosión, en alto riesgo se encuentra las zonas que están aguas abajo de los lugares de formación de bancos de arena ya que estos puntos al ser continuamente explotados, se tienden a amentar el gradiente y la erosión en estos sectores es más prominente, tal como se valida en las visualizaciones, actualmente en las dos zonas mostradas en la siguiente figura existe una continua explotación desde hace más de 40 años, los que según los resultados generados, se ve una tensión de corte media y alta justo en los sectores aguas abajo de los puntos de extracción de materiales de arrastre.

\section{Conclusiones}

La estimación de formación de bancos de arena a partir del cálculo de transporte de sedimentos o carga de fondo por medio de la relación de Meyer- Peter \& Muller y la función de Shields permite reconocer matemáticamente el estado real del lecho del río que se quiere estudiar, tal como se evidencia en los resultados obtenidos, el Río Cauca debido a sus características fluviales y topográficas cuenta con un alto potencial de transporte y acumulación de sedimentos. En primera instancia al reconocer la carga de fondo se estableció las zonas aptas para la acumulación, lo que después se validó con los resultados obtenidos en la capacidad de estas zonas para la formación de bancos de arena, 
planteamiento que fue validado teniendo en cuanto a situaciones actuales (2014) que presenta el río basado en las visualizaciones disponibles en Google Earth.

En los resultados obtenidos se tuvo gran validez ya que en gran parte, estos coincidieron con situaciones actuales (2014) presentes sobre el lecho del Río Cauca, con relación a la tensión de corte o erosión sobre el lecho se validó en gran parte la bibliografía consultada ya que según Ramírez et al. (2009); Shaw et al. (1994); Moghadam et al. (2013) y Lorang et al. (2005) aguas abajo de los sitios de extracción de materiales de arrastre por el aumento de la turbiedad y de los sedimentos suspendidos se iba aumentar la erosión del lecho del río, tal como se evidencia en la Figura 13 correspondiente a la tensión de corte o erosión, respecto a las zonas de continua extracción de agregados por el sector constructivo, en relación al cálculo de la potencia del río los resultados mostraron veracidad la cual se mantiene hasta la fecha debido a la constancia en la extracción de sedimentos, tal como ve en la Figura 12 de los resultados de potencia para el tramo seleccionado se caracteriza por una potencia media y en algunos sectores alta esto se debe a que la extracción de material aguas abajo ocasiona un aumento gradiente hidráulico y de la velocidad de flujo, aumentando la potencia en estos sitios produciendo mayor transporte de sedimentos y desgaste sobre el lecho de acumulación.

Debido a la gran coherencia en los resultados obtenidos y que aun a la fecha se tenga validez en los mismo se concluye que en relación a al reconocimiento del lecho del río en un tiempo aproximado de 10 años no se han presentado cambios y aun se pueden realizar análisis con los resultados obtenidos para la fecha actual (2014), de igual manera se reconoce que una menor diferencia en la temporalidad de la captura de los datos permitirá un mejor reconocimiento del lecho y hasta se podría pronosticar eventualidades partiendo de la correlación obtenida.

\section{Referencias bibliográficas}

Cámara Colombiana de la Construcción. CAMACOL. (2013). Estudio de oferta y demanda de vivienda. Cali, Colombia. https://bit.ly/39Vq8a7

Ramírez, C., Bocanegra, R., Santacruz, S., Quintero, H., Sandoval, M. C. (2009). Metodología para estimar los volumenes maximos de explotacion de materiales de arrastre. Ingenieria y Competitividad, 11(2), 79-93. https://doi.org/10.25100/iyc.v11i2.2463 
Church, M. (2006). Bed Material Transport and the Morphology of Alluvial River Channels. Annual Review of Earth and Planetary Science, 34, 325-353. https://doi.org/10.1146/annurev.earth.33.092203.122721

Corporación Autónoma Regional del Valle del Cauca. (1999). Propuesta de restriccion de las explotaciones de materiales de arrastre en algunos rios del departamento del Valle del Cauca. Santiago de Cali.

Corporación Autónoma Regional del Valle del Cauca. (15 de julio de 2008). Proyecto de Modelación del Río Cauca PMC. http://www.cvc.gov.co/cvc/Mosaic/

Ferguson, R. (2008). Gravel-bed rivers at the reach scale. En H. Habersack, H. Piegay y M. Rinaldi (Eds.), Gravel-Bed Rivers VI: From Process Understanding to River Restoration (pp. 33-53). Elsevier Science.

Lorang, M. S., Whited, D. C., Hauer, F. R., Kimball, y Stanford, J. A. (2005). Using Airborne Multispectral Imagery to Evaluate Geomorphic Work across Floodplains of Gravel-Bed Rivers. Ecological Applications, 15(4), 1209-1222.

Rezaei Moghadam, M. Z., Mohammadfar, A., y Valizadeh Kamran, Kh. (2013). Changes Detection and identification of erosion risk areas of Aji Chay River between Khaje to Vanyar. Geography and Environmental Planning Journal, 23(4), 1-14.

Shaw, P., Shick, A., y Hassan, M. (1994). Bedload Sediment Transport in the Sand Rivers of Botswana. Botswana Notes and Records, 26(1994), 115-127.

Zeiss. (1996). DEUSTSCHER KALIBRIERDIENST (DKD). Calibration certificate. Oberkochen, Alemania: Carl Zeiss Servicebereich Qualitat MeB-/ Kalibrierzentrum. 